\title{
Gesture Recognition Using Commodity RGB-D Sensor for Imitation Learning Platform for Children with Autism
}

\author{
Esubalew Bekele, Jake Bumpus, Shuvajit Das, Julie Crittendon, \\ Zachary Warren, and Nilanjan Sarkar \\ Vanderbilt University, 518 Olin Hall 2400 Highland Ave Nashville / TN 37212, USA, \\ University of Michigan - Ann Arbor, MI 48109 USA \\ \{esubalew.bekele, jacob.m. bumpus, julie.a.crittendon, \\ zachary.warren, nilanjan.sarkar\} @vanderbilt.edu, shudas@umich.edu
}

\begin{abstract}
Autism Spectrum Disorders (ASD) is characterized by profound impairments in social interaction and communication. Children with ASD have deficits in core areas of social interactions such as gesture imitation. Gesture imitation is one of the early developed social communication skills and is thought to be linked with concurrent as well as later complex social skills such as language development, play and joint attention skills. Thus early identification of such a deficit and providing appropriate intervention regarding gestural imitation skills are quite important. This work, which is a part of a larger study that aims at building a gesture imitation intervention platform for children with ASD, leverages the intrinsic interest of the children with ASD in robotic technology. In this paper, we discuss the part of the robot-mediated intervention system that deals with gesture recognition and present preliminary recognition results.
\end{abstract}

Keywords: Gesture recognition, hidden Markov models, autism intervention, adaptive interaction, RGB-D sensor, assistive robotics.

\section{Introduction}

Young children with autism spectrum disorders (ASD) display profound impairments in social communication and atypical patterns of behaviors [1] such as delayed language development, pretend play, joint attention and imitation [2]. Imitation impairments were associated to co-occur with other impairments concurrently or affect later developmental outcomes [3,4] including language development, pretend play, and joint attention for children with ASD [3,5]. Children with autism lack the use of gestures, which are related to joint attention and expression of affective states, than typically developing children [6]. Therefore improvement in gesture imitation may translate into improvement in complex social and communication skills. Ingersoll et al. [6] argued that very few research has been done in teaching imitation of meaningful gestures to children with autism and most research focuses on directly commanding the children to do a particular isolated gesture or sequence of gestures 
which limits the children from learning novel gestures and hinders generalization. They suggest a more naturalistic intervention called reciprocal imitation training (RIT) that was aimed at teaching imitation skills to young children with autism within an ongoing play interaction. It was reported that RIT not only improved the gesture imitation capability but also helped with increase in pretend play skills. However, intensive behavioral interventions such as RIT require trained behavioral therapists and entail repetitive intervention over an extended period of time and hence, are not available to the wider ASD population. Due to the repetitive nature of the intensive behavioral therapy, robotic technology is suited for such service delivery. It is documented that children with ASD are interested in robots and technology and may prefer to play with a robot than humans $[7,8]$. Robot-mediated joint attention therapy was piloted in earlier study and found that it created increased engagement while performing as well as a human therapist [9]. Imitation learning and robot programming by demonstration have been an active research area in the past decades [10,11]. Also, traditional gestural imitation learning intervention for children with autism have been an active area of research separately [6]. In recent years, applications of robot-based imitation learning for therapy have also been investigated $[12,13]$.

The overall objective of our project is to develop an innovative robot-mediated imitation learning intervention environment for children with autism based on RIT. The overall system will have two main subsystems: gesture recognition of the participants by the robot, and demonstration of correct gestures and providing feedback by the robot. The contribution of this paper is in the development and evaluation of the gesture recognition part of the system. The recognized gestural data has been mapped to the robot workspace for validation.

\section{General System Overview}

The robot-mediated imitation learning platform in its current state is composed of the Microsoft Kinect, NAO - a humanoid Robot, and a gesture recognition module. The current system that recognizes a gesture demonstrated by the child and maps it to the robot is shown in Fig. 1 (left). The gesture recognition module consists of data preprocessing, gesture training, gesture recognition, and workspace mapping components.

\subsection{The Humanoid Robot, NAO}

The robot used in this project is the humanoid robot NAO (www.aldebaranrobotics.com). Its software was built around a programming architecture with a network broker called NAOqi. Task specific modules can be written and attached to the broker either inside the robot's operating system locally or remotely. This broker process, which is based on the Simple Object Access Protocol (SOAP) specification, allows extending NAO's capabilities via distributed system architecture. We leveraged this ability and run most of the computationally intensive tasks of the system such as the gesture recognition component on separate machines. 


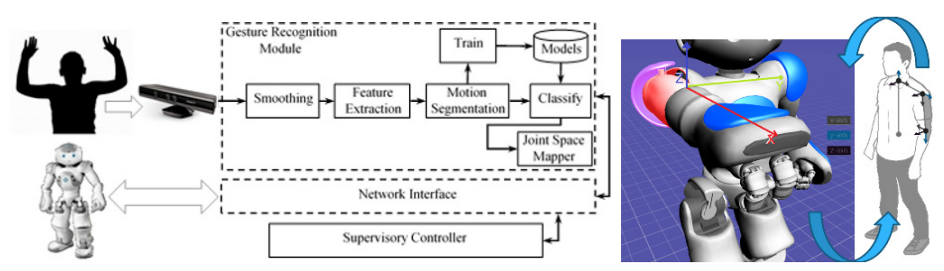

Fig. 1. Overall system diagram (left), and joint mapping (right)

\subsection{Gesture Monitoring with Kinect}

The Microsoft Kinect skeletal tracker can track up to two humans in its field-of-view and provides the ability to fit a 20 -joint skeletal model to each user that is being currently tracked. The raw skeletal joints data were first filtered to eliminate spurious data outside of the workspace limits, data that produces jerky motion as well as noise. The data were filtered both in time and space and a time median filter was used to smooth the trajectories of a particular gesture demonstration. We have extracted various features from the raw data such as relative distance of the joint from a shoulder center reference for each joint involved in a particular gesture and then applied a forward feature selection procedure called sequential forward selection (SFS). A direct joint to joint mapping between the robot and the human space was used as shown in Fig. 1 (right) to demonstrate the recognized gestures.

\subsection{Gesture Recognition with HMM}

A gesture is inherently composed of sequence of primitive action sequences that can be modeled efficiently by the Hidden Markov Model (HMM) [14]. Given: $N$, the number of states in a model, $M$, the number of distinct observable output symbols per state, $\mathrm{A}=\left\{a_{i j}\right\}$ (the transition from state $i$ to $j$ ), the state transition probabilities, $\mathrm{B}=$ $\left\{b_{j}(k)\right\}$ (symbol $V_{k}$ given state $\mathrm{j}$ ), the observation symbol probability distribution, and the initial state $\Pi=\left\{\pi_{i}\right\}$, a discrete HMM can be defined as $\lambda=(\mathrm{A}, \mathrm{B}, \Pi)$. HMM models are generated from a sequence of training dataset. The recognition phase evaluates the observation likelihood of the observation sequence given the model, $P(O / \lambda)$. The solution to the training problem is in the form of locally maximizing the $P(O / \lambda)$. The feature vector stream was first clustered and labeled into $\mathrm{M}$ number of output symbols before the sequence is fed to training. In this work, we used the kmeans clustering. We have selected two sets of five gestures. The first gesture set is composed of symbolic gestures including moving the arm in a circle, 'come here', move the arm in a rectangle, swipe, and wave. The second set includes gestures which are part of the unstructured imitation assessment (UIA) imitation action gestures from Ingersoll et al. [2]. We have recorded 30 instances of each gesture (with two different people at different poses) and used 20 examples as the training set and the remaining 10 as the testing set. 


\section{Results}

To evaluate the performance of the gesture recognition system we first used the 3-D trajectory of the end effector (wrist). Then from a high dimensional feature vector (105 features), we selected the features for the wrist and performed SFS. We used two degrees of freedom left-to-right HMM models with 8 hidden states and 12 output with 3 dimensional feature vectors taken at a time. There were two sets of gestures. Fig. 3 shows segmented two dimensional three gesture trajectories from each set. Different colors indicate different segments of each gesture that are grouped for a particular output symbol in the vector quantization stage.

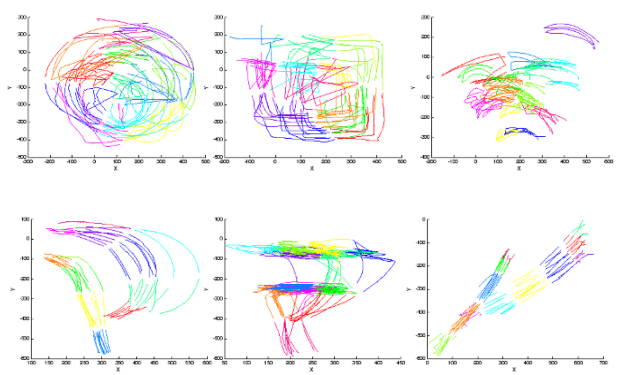

Fig. 2. Top: Set 1 (Circle, Rectangle, and Wave). Bottom: Set 2 (hands on head, move fingers in circle and point)

The average recognition rate for gesture set 1 was $68 \%$ using raw trajectory while gesture set 2 resulted in average recognition rate of $96 \%$. So, gesture set 2 was selected for further SFS analysis. The recognition results using the individual feature categories in the first level of the SFS process was more than $95 \%$ for all the 5 gestures and that of final winner feature combination was $100 \%$. The best feature set was found to be combination of Euler angles, polar, Cartesian and spherical velocities, and relative distance of the wrist relative to the shoulder.

\section{Discussion and Conclusion}

The recognition system was validated for known gesture sets and was able to recognize them accurately. However, there were several limitations of the current system including inability to handle novel gestures. In the future, the system will be expanded to include an intelligent supervisory controller that is able to perform in a bi-directional manner to make the robot-mediated therapy resemble closely to that of the human therapist by demonstrating a gesture to the child. In the case of partial or complete success, the robot should be able to give reinforcement contingent upon success and imitate back the child in the case of contingent imitation. Isolating gesture from a movement stream, transferring summery information about the gesture using continuous HMM, mapping it to the robot workspace in a generalized manner and completing incomplete attempt of a gesture by the child are also part of future 
extensions of the system. Gesture sequence matching algorithm in time domain such as dynamic time warping could also be employed to make the sequences equal length before clustering.

\section{References}

1. Diagnostic and Statistical Manual of Mental Disorders: Quick reference to the diagnostic criteria from DSM-IV-TR, American Psychiatric Association, Amer. Psychiatric Pub. Incorporated, Washington, DC (2000)

2. Ingersoll, B., Meyer, K.: Examination of correlates of different imitative functions in young children with autism spectrum disorders. Research in Autism Spectrum Disorders 5(3), 1078-1085 (2011)

3. Stone, W.L., Yoder, P.J.: Predicting spoken language level in children with autism spectrum disorders. Autism 5(4), 341-361 (2001)

4. Stone, W.L., Ousley, O.Y., Littleford, C.D.: Motor imitation in young children with autism: what's the object? Journal of Abnormal Child Psychology 25(6), 475-485 (1997)

5. Rogers, S.J., Hepburn, S.L., Stackhouse, T., Wehner, E.: Imitation performance in toddlers with autism and those with other developmental disorders. Journal of Child Psychology and Psychiatry 44(5), 763-781 (2003)

6. Ingersoll, B., Lewis, E., Kroman, E.: Teaching the imitation and spontaneous use of descriptive gestures in young children with autism using a naturalistic behavioral intervention. Journal of Autism and Developmental Disorders 37(8), 1446-1456 (2007)

7. Diehl, J.J., Schmitt, L.M., Villano, M., Crowell, C.R.: The clinical use of robots for individuals with Autism Spectrum Disorders: A critical review. Research in Autism Spectrum Disorders 6(1), 249-262 (2011), doi:10.1016/j.rasd.2011.05.006

8. Scassellati, B., Admoni, H., Matarić, M.: Humanoid Robots for Use in Autism Diagnosis/Research. Annual Review of Biomedical Engineering 14(1), 275-294 (2012)

9. Bekele, E., Lahiri, U., Swanson, A., Crittendon, J., Warren, Z., Sarkar, N.: A Step Towards Developing Adaptive Robot-Mediated Intervention Architecture (ARIA) for Children With Autism. IEEE Transactions on Neural Systems and Rehabilitation Engineering 21(2), 289-299 (2012)

10. Schaal, S.: Is imitation learning the route to humanoid robots? Trends in Cognitive Sciences 3(6), 233-242 (1999)

11. Calinon, S., D'halluin, F., Sauser, E.L., Caldwell, D.G., Billard, A.G.: Learning and reproduction of gestures by imitation. IEEE Robotics \& Automation Magazine 17(2), 44-54 (2010)

12. Duquette, A., Michaud, F., Mercier, H.: Exploring the use of a mobile robot as an imitation agent with children with low-functioning autism. Autonomous Robots 24(2), 147-157 (2008)

13. Fujimoto, I., Matsumoto, T., De Silva, P.R.S., Kobayashi, M., Higashi, M.: Mimicking and Evaluating Human Motion to Improve the Imitation Skill of Children with Autism Through a Robot. International Journal of Social Robotics, 1-9 (2011)

14. Rabiner, L.R.: A tutorial on hidden Markov models and selected applications in speech recognition. Proceedings of the IEEE 77(2), 257-286 (1989) 